\title{
An Iot based Temperature Monitoring System for Industries and Transportations
}

\author{
Revathi R, Suganya M, Alan Ronald Arasu M.C, Balakrishnan S, Lokesh L
}

\begin{abstract}
Temperature that can vary as high or low especially in Industries will cause discomfort to products and organization. So, we use Temperature sensors to monitor and measure the temperature at industries. These sensors are fixed into a component which tends to be problematic due to overheating issues. The sensors use a GSM module which constantly sends the temperature updates of the machine and surroundings to your Personalized Devices. This project not only tells the temperature in the industry and machines but also changes the temperature according to the organization by turning on the humidifiers or Extinguisher around the area. This can also send Emergency signals to the fire department if there is a serious fire. These devices are embedded with GSM module So these can also be used in Transport Systems so it can track the location and monitor the temperature around it at the same time.
\end{abstract}

Keywords: IoT, temperature monitoring and control, Production Industry, GPS tracking

\section{INTRODUCTION}

A s industries continue to grow and expand, so is the effort required to maintain them, transport of the products, their storage, etc. The power of IoT can be used to simplify these growing demands of maintenance. The IoT based temperature monitoring system can be used alongside machines to monitor and control their temperature 24/7 and raise an alarm should there be any discrepancy thus preventing any accidents and ensuring that the temperature conditions are optimal for production. It can also be used inside transport vehicles to track their location \& monitor the Temperature of the Container es to prevent spoilage of products.

Revised Manuscript Received on April 13, 2020.

* Correspondence Author

Revathi R*, Assistant Professor, Department of Computer Science \& Engineering, Jeppiaar Institute of Technology, Sriperumbudur, India.

Email: revathi85it@yahoo.co.in

Suganya M, Assistant Professor, Department of Computer Science \& Engineering, Jeppiaar Institute of Technology, Sriperumbudur, India Email: suganyam@jeppiaarinstitute.org

Alan Ronald Arasu M.C, Department of Computer Science \& Engineering, Jeppiaar Institute of Technology, Sriperumbudur, India. Email: alanronald55@gmail.com

Balakrishnan S, Department of Computer Science \& Engineering, Jeppiaar Institute of Technology, Sriperumbudur, India. Email: balakrish1998s@gmail.com

Lokesh L, Department of Computer Science \& Engineering, Jeppiaar Institute of Technology, Sriperumbudur, KA, India. Email: lokeshmarison0019@gmail.com

(C) The Authors. Published by Blue Eyes Intelligence Engineering and Sciences Publication (BEIESP). This is an open access article under the CC BY-NC-ND license (http://creativecommons.org/licenses/by-nc-nd/4.0/)

\section{INTERNET OF THINGS}

IoT is a network of objects connected with the Internet that collects and exchange data. IoT is often known by the physical objects connected with the network. Objects can be anything like devices, vehicles, Environment or anything which are embedded with Internet connectivity, sensors, electronics and software which allows the objects to transfer or collect data. It gives a capability to the "Things" to transfer data without any human to human or computer interaction. In IoT, "Things" are often referred to many things which can be a built-in sensor for vehicles, heart monitor implants, or even biochip transponders for farm animals. It is a collective system of computing devices that can sense their environment through sensors and share data over a network without requiring any human interaction. It is a modern technology that can sense, process, store and transmit data. This data can be further used for real-time analytics and machine learning purposes. Due to its potential, IoT's applications continue to grow every day ranging from home appliances to automobiles enabling customers to control devices without manual intervention.

\section{METHODOLOGY}

In this Prototype, we are going to

$>$ Analyze the temperature of the machine by gathering the data from the sensor and send them to a private cloud to constantly monitor it.

$>$ Track the location of the transport using our GPS module Embedded inside the module.

$>$ Control the temperature of the Machine by enabling cooling devices through Mobile Application.

$>$ Alert the Authorities in case of any Sudden fire Accidents.

$>$ If the Users doesn't Respond to the Alert a period, it enables Automatic Fire/Heat Suppression System by Enabling the nearby cooling devices.

\section{A. Existing System}

Machines in large scale industries had to be monitored Individually with separate workers for Each machine. Even though workers are Assigned it can't be easily monitored and reported properly to the Authorities. In the case of Fire Emergency, humans can't easily handle that kind of situation. Human lives are more important than machines, in that case, to overcome this we have designed a System which does those things for us without involving human life in danger.

Published By:

Blue Eyes Intelligence Engineering \& Sciences Publication

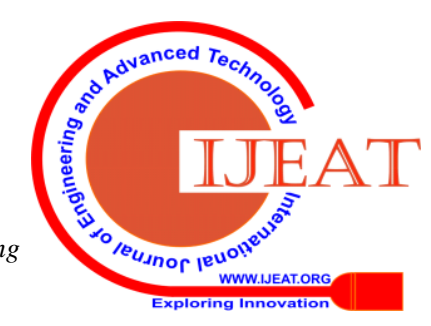




\section{B. Proposed System}

Our proposed system, A Temperature Sensor is placed in the machine itself which constantly monitor the temperature and humidity of the machine and the around it. The gathered Data is constantly noted in the database. When the System/Machine Crosses the Recommended/Critical Temperature, it Alerts the Admin to enable control measures for the temperature spike. If the Admin is Unavailable, the System Automatically turns on the Cooling System. In Case of sudden fire, the sensor node automatically alerts the fire department by providing with the Location.

\section{WORKING}

The working process of our System has been divided into five different phases.

\section{A. Sensing Phase}

In this phase the Sensors Gathers data such as temperature, humidity and location form the Machine and from the environment using DHT 22 (Temperature sensor) and Neo-6M (GPS) module. The Sensed Analog data is Converted to Digital in the sensor by inbuild chips and the digital data is sent to the Microcontroller Unit.

\section{B. Processing Phase}

The Node MCU consists of an ESP8266(LX106) Processor which can be programmed using Sketch. The sketch is a unit code that is uploaded to the Arduino board. Arduino IDE is used to compile the Sketch code and upload it into the processor. The Microprocessor is programmed to collect the data from different sensors and process the data for Transmission. The Critical Point Alert notification can be set in this phase itself.

\section{Communicating Phase}

In this phase, the processed data is sent to a Cloud Server Database via SIM900A(GPRS). The server Database which keeps a log of the data and updates it to the User's personalized devices simultaneously. So, the user can check the status of the machine/Vehicle any time they want.

\section{Activation Phase}

This phase consists of an Emergency trigger Alert from the node MCU. When the increase in Temperature is sensed by the Sensor it is triggered by the Program and Automatically send Call/text Alerts the User to make Control measures.

\section{E. Response phase}

When User Gets the Alert on temperature Spike the System request Control measures to enable Cooling System. The response from the user's App will reach the Node MCU to enable the Cooling System around it through Wi-Fi. If the user is unavailable, then the System automatically enables the emergency Cooling System.

In Case of Sudden Fire, the Node will also Intimate the nearby Fire Station with the exact point of location Automatically. within a period if the user goes offline.

The user can keep track of the Transport vehicles with the help of (Neo 6m) GPS module embedded with our System and control the Environment inside the Vehicle.

\section{HARDWARE USED}

\section{A. NodeMCU}

NodeMCU is like an Arduino device. The main component of Node is ESP8266. Node microprocessor has Programmable pins and a built-in Wi-Fi. This MCU is powered through a Micro USB port. It can be programmed through multiple programming environments. NodeMCU is open-source and Its hardware design is open so that anyone can edit, modify, build. Node Microprocessor Kit or a board which consist of ESP8266 enabled chip. ESP8266 is a low-cost Wi-Fi-enabled chip which transfers data using TCP / IP protocol. This Microprocessor uses a flash SPIFFS (Serial Peripheral Interface Flash File System)in the module itself. The NodeMCU is implemented in C.

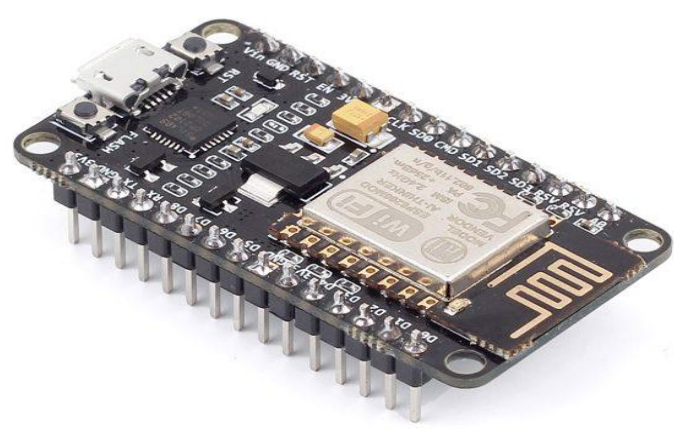

Fig. 1. Node MCU

\section{B. DHT 22}

The DHT22 is a digital temperature and humidity sensor. DHT22 is a low-cost sensor. It uses a capacitive humidity sensor and an NTC temperature sensor or a thermistor which measures the surrounding environment and sends the digital signal to the Data pin. The Analog data from the sensors are converted to digital data by a basic Chip. The DHT22 calculates the humidity by measuring the electrical resistance between two electrodes. It has a sampling rate of $0.5 \mathrm{~Hz}$ which is for every two seconds one reading can be acquired. The temperature measuring range is from -40 to +125 degrees Celsius with +-0.5 degrees accuracy and the humidity measuring range is from 0 to $100 \%$ with $2-5 \%$ accuracy. The Range is higher than DHT11 Sensor because in this project the sensor needs to withstand a ton of temperature change.

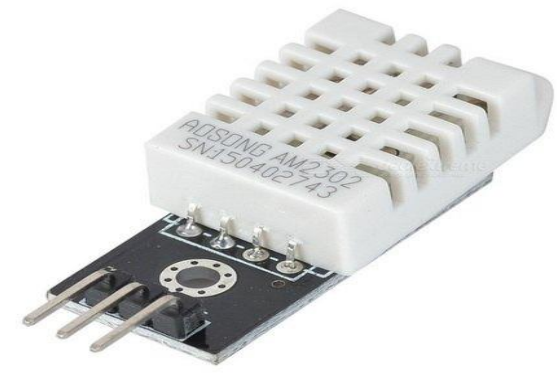

Fig. 2. Digital Humidity and Temperature (DHT22) Sensor

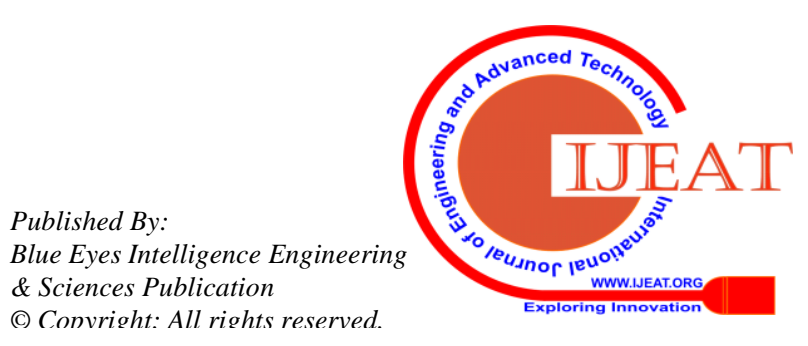




\section{SIM 900 A}

SIM900A is a reliable wireless module. It is an ultra-compact module and has a Dual Band GSM/GPRS which works with frequencies of 900/1800MHz. SIM900A can find these two bands automatically. It requires only $5 \mathrm{~V}$ input. The AT Commands in the IDE can be used to set the frequency band. The baud rate is of SIM 900A is configurable from 1200 to 115200 in the IDE with AT command. This Modem is Embedded with internal TCP/IP stack which gives you internet connectivity through GPRS. It is a Dual-band GSM/GPRS solution in an SMT module with a powerful AMR926EJ-S Core single-chip processor. This module is used to send data processed from node MCU to the server and receive the control commands from the user.

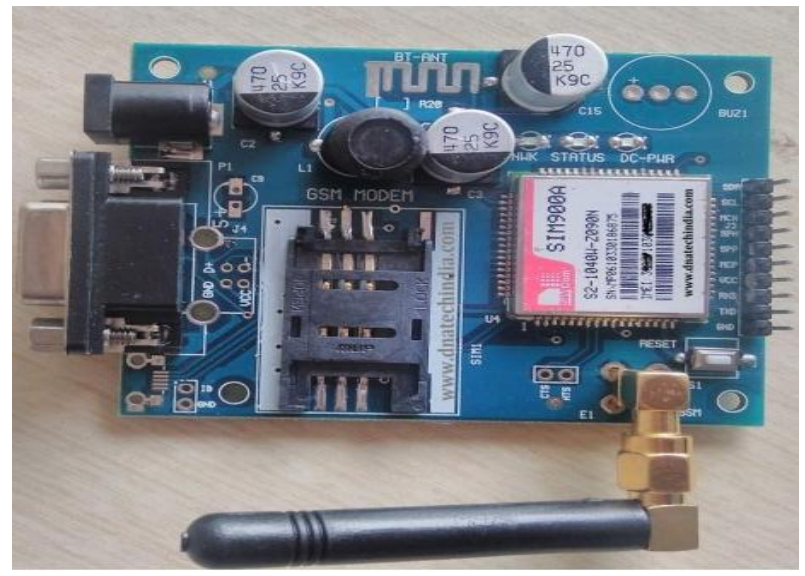

Fig. 3. Global System for Mobile communications.

\section{NEO 6M}

The NEO-6M global positioning system (GPS module) is a high-performance and also a cost-effective GPS module with an onboard memory chip and aceramic antenna and it has a high sensitivity for indoor applications. It also has EEPROM for storing configuration settings. The NEO-6M works with 3.3V to 5V DC input. The NEO-6M GPS module has a strong satellite search capability. With the integrated signal and power indicators and status of the module can be monitored in the module itself. GPS takes time to find the Accurate location by Triangulating with 3 satellites.

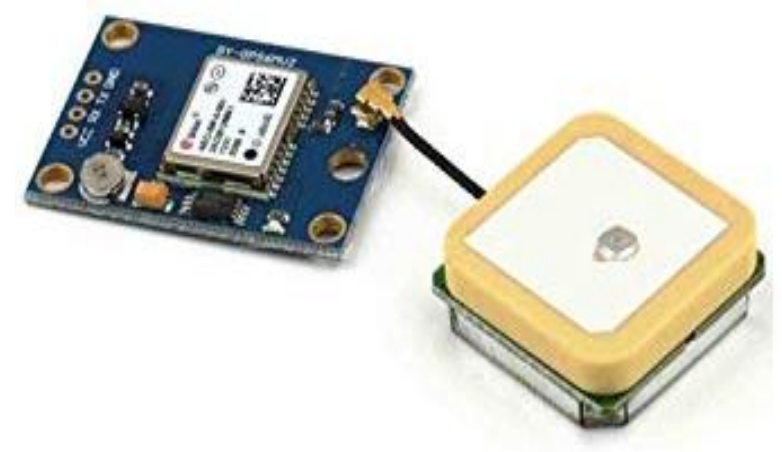

Fig. 4. Global Positioning System.

\section{ARCHITECTURE}

This Diagram of our project will clearly explain the whole workflow of our system. Data are sensed by the sensor and Stored it into the database, the mobile devices get the data from the database and display it as a chart for temperature and map for location. The node can communicate with nearby cooling devices. Emergency Alerts to the Fire depart is given with the location of the machine in case any Sudden fire incident.

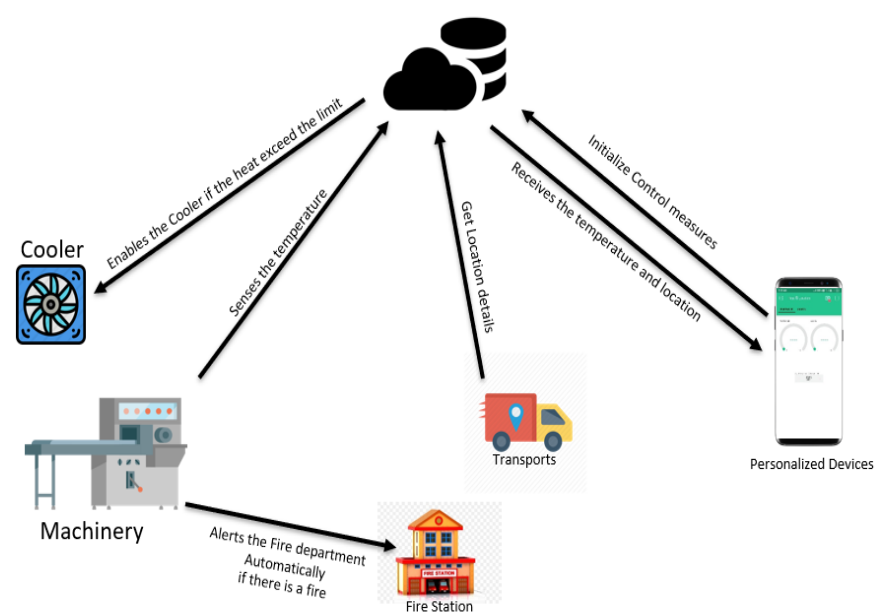

Fig. 5. Architecture

VII. RESULT AND OUTPUT

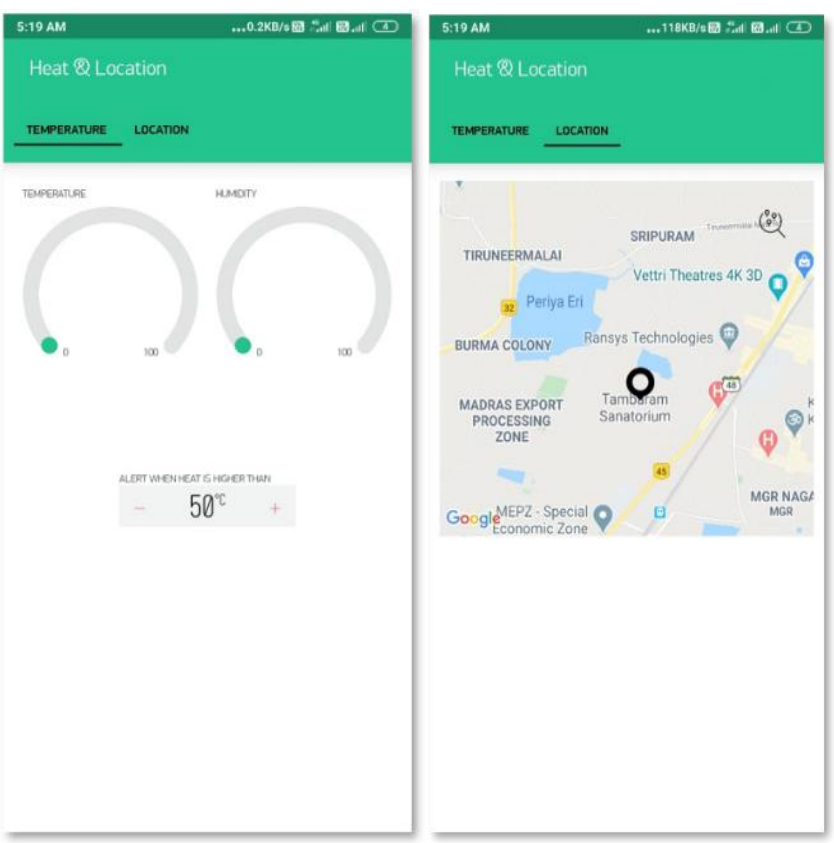

Fig. 6. Mobile Application to monitor and Control the temperature

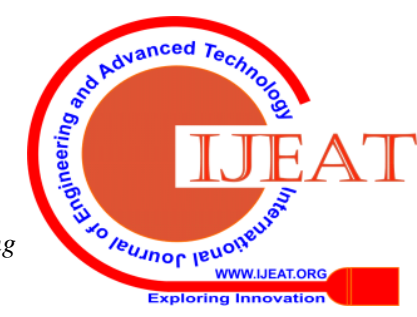




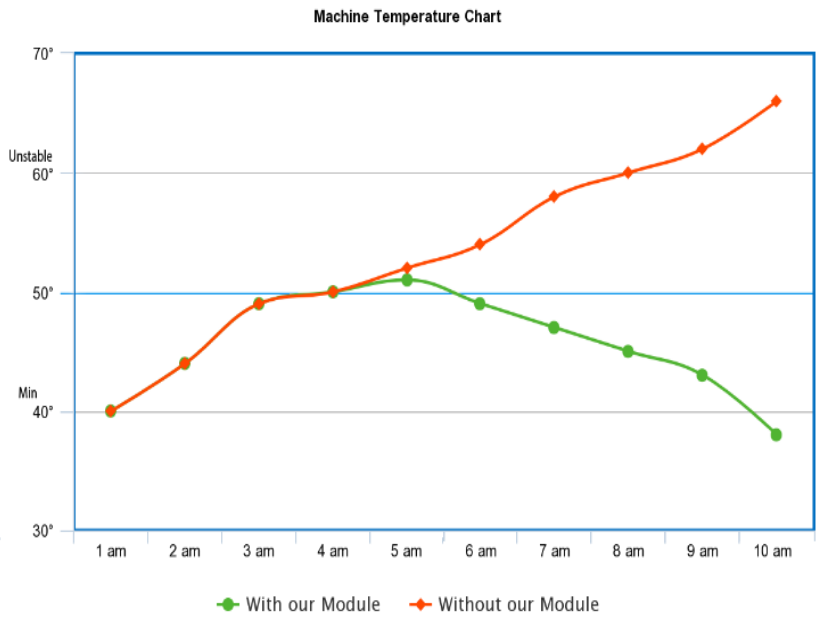

Fig. 7. Temperature Chart of a machine with and without our module.

\section{CONCLUSION}

Our prototype is very much useful in Large scale Industries and Transports. This Avoids fire Accidents caused by overheating of the machine and reduces the heat damage in the products.

\section{FUTURE WORK}

Our Prototype can use only a battery as the Power Source. So, the energy consumption of the node needs to be less to have a long lifetime. The future work of this project will be to reduce the power consumption of the System. To achieve this, the hardware needs to be modified to send data only whenever necessary, and from the software side, power-saving algorithms need to be added to reduce the power consumption of the System.

\section{REFERENCES}

1. Huan-Chieh Chiu, Cheng-Ying Chou, Meng-Fu Chen, Kai-Sheng Tseng, Yu-Cheng Yan, Joe-Air Jiang (2019). “An IoT-based Temperature Monitoring System for Underground Cable Tunnels" of Grand International Conference and Exposition Asia, IEEE PES GTD.

2. Dan Chia-Tien Le, Amir Atabekov, Marcel Starosielsky, Jing Selena He (2015). "Internet of Things-Based Temperature Tracking System" in Computer Software and Applications Conference (COMPSAC), IEEE Annual International.

3. Chien-Kuo, Chang Wu and Ruay-Nan (2011). "Use of partial discharges as an online monitoring system for underground cable joints," in IEEE Transactions on Power Delivery.

4. Aniket Parab, Medha Kulkarni, Vinod Choudhary, Neetesh Jha, Satyajit Bhapkar (2016). "Design and Implementation of Wi-Fi-based Smart Home System" in International Journal of Engineering and Computer Science, Mumbai, 400022, India, vol 5, no 02.

5. Rahul Johari, Alok Kumar Gupta, (2019). "IOT based Electrical Device Surveillance and Control System of Internet of Things": Smart Innovation and Usages (IoT-SIU), International Conference On IEEE.

6. Abdur Rahim Biswas, Raffaele Giaffreda (2014). "IoT and cloud convergence: Opportunities and challenges of the Internet of Things (WF-IoT)", IEEE World Forum on IEEE.

7. Shyamambika, N. and Thillaiarasu, N., (2016). "A survey on acquiring the integrity of shared data with effective user termination in the cloud" in Intelligent Systems and Control, International Conference on IEEE.

8. Sunil Kumar, Subhranil Som Khatri, Rahul Dagar, (2018). "Smart Farming - IoT in Agriculture" in International Conference on Inventive Research in Computing Applications (ICIRCA), International Conference on IEEE.
9. Lalbihari Barik (2019). "IoT based Temperature and Humidity Controlling using Arduino and Raspberry Pi” of International Journal of Advanced Computer Science and Applications, Vol. 10, No. 9.

10. Bing Jiang, A.P. Sample, A.V. Mamishev, R.M. Wistort (2005). "Autonomous robotic monitoring of underground cable systems" at 12th International Conference on Advanced Robotics, ICAR '05. Proceedings, Seattle, WA, USA, pp. 673-679.

11. ChenthurPandian, S and Thillaiarasu, N. (2016). "Enforcing security and privacy over the multi-cloud framework using assessment techniques" at Intelligent Systems and Control (ISCO), International Conference on IEEE.

12. Asha Rawat, Gaurav Waradkar, Vinay Maitry, Hitesh Ramina, Tejasvi Ansurkar, Parth Das (2016). "Automated room light controller with visitor counter", in Imperial Journal of Interdisciplinary Research.

13. He Shabai, per Ängskog, Javier Ferrer-Coll, Peter Stenumgaard and José Chilo (2012). "Analysis of wireless communications in underground tunnels for industrial use," in IECON 2012 - 38th Annual Conference on IEEE Industrial Electronics Society, Montreal, QC, Canada.

14. S.M. Kusuma, Sagar KN Vinay (2015). "Home Automation Using Internet of Things" in the International Research Journal of Engineering and Technology (IRJET).

15. Kirkland, J W. and Lyle, R. (2011). "An Accelerated Life Test for Evaluating Power Cable Insulation" on IEEE Transactions on Power Apparatus and Systems, vol. PAS-100, no. 8, pp. 3764-3774.

\section{AUTHORS PROFILE}

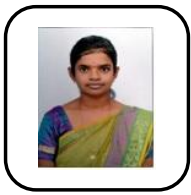

Ms. R Revathi is currently an Assistant Professor in the Department of Computer Science and Engineering at Jeppiaar Institute of Technology, Chennai. She has 7 Years of Teaching \& 1 year of Industrial Experience. She secured distinction in both UG and PG degree. She has handled various Computer science \& Information Technology subjects like Computer Architecture, Mobile Communication, Design Analysis \& Algorithm, Object Oriented Programming, Software Project \& Management, Object Oriented Analysis \& Design, Software Testing, etc. She has published papers in Scopus Indexed Journals and also presented 4 papers in various National and International Conferences. She has completed 3 online courses and certified has 5S-Internal Auditor.

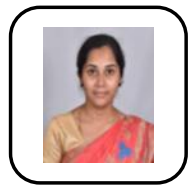

Ms. M Suganya is currently working as an Assistant Professor in the Department of Computer Science and Engineering at Jeppiaar Institute of Technology, Chennai. She has 1 year and 8 months of Teaching \& 2+ years of Industrial Experience. She was awarded 6th rank in Anna University rank list among 984 candidates in her PG degree. She has published papers in Scopus Indexed Journals and also presented $10+$ papers in various National and International Conferences. She has good exposure to programming skills and Cloud technologies. She has been awarded "Young Research Engineer" award for carrying out vibrant activities related to small satellites, involving students and academic institutions under the banner of UNISEC India in the areas of Nanosats, Cansats and Cubesats. Her area of Interest is Cloud Computing, in which she's doing her research work.



Alan Ronald Arasu M.C is currently pursuing his bachelor's degree in the field of Computer Science and Engineering at Jeppiaar Institute of Technology, Kanchipuram, Tamil Nadu, India. He did his schooling in Chennai. He is particularly interested in IoT, Web Designing, App Development Cloud computing, etc.

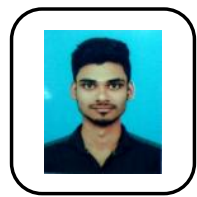

Balakrishnan S is currently pursuing his bachelor's degree in the field of Computer Science and Engineering at Jeppiaar Institute of Technology, Kanchipuram, Tamil Nadu, India. He did his schooling in Chennai. He is particularly interested in Algorithm and Data Structures, Java.

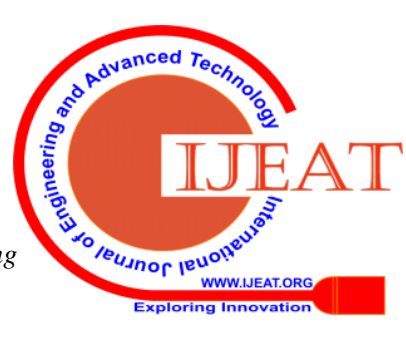


Lokesh $\mathbf{L}$ is currently pursuing his bachelor's degree in the field of Computer Science and Engineering at Jeppiaar Institute of Technology, Kanchipuram, Tamil Nadu, India. He did his schooling in Kanchipuram. He is particularly interested in Math, Analytical and Problem-Solving Skills.

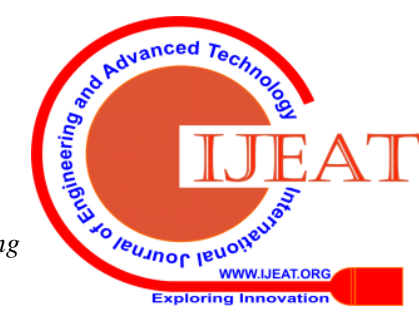

